\title{
O USO DE APLICATIVOS PARA AUXILIAR NO DESENVOLVIMENTO DE CRIANCAS COM TRANSTORNO DO ESPECTRO AUTISTA
}

The use of applications to auxiliate the development of children with autistic spectrum transtorn

\author{
Maíra Carla Moreira Aragão* \\ João Batista Bottentuit Júnior \\ Lívia da Conceição Costa Zaqueu ${ }^{* * *}$
}

\begin{abstract}
RESUMO: O presente artigo visa mostrar as ferramentas tecnológicas que podem ser utilizadas a favor de crianças com Transtorno do Espectro Autista como facilitadoras no desenvolvimento de diversas habilidades. A pesquisa de cunho bibliográfico analisou quatro aplicativos disponíveis no mercado tecnológico destacando o funcionamento, os objetivos, as possibilidades e sua disponibilidade. Ao final da pesquisa, realizamos um quadro comparativo dos aplicativos evidenciando as características distintas e diferentes entre eles. Concluímos que o aplicativo "ABC Autismo" possui maior abrangência comercial, com mais de cem mil downloads e apresenta-se em diferentes versões: inglês, português e espanhol. $\mathrm{O}$ aplicativo "AUTApp" contempla melhor as necessidades específicas relacionada às emoções. $\mathrm{O}$ aplicativo "TEO" diferencia-se por apresentar além das habilidades básicas, atividades de desenvolvimento cognitivo. O aplicativo "As Descobertas de Albert" possui menor capacidade memorial e é o único que pode ser encontrado em Android e IOS.
\end{abstract}

PALAVRAS-CHAVE: Dispositivos móveis. Aplicativos. Transtorno do espectro autista

\begin{abstract}
This article aims to show the technological tools that can be used to help children with Autism Spectrum Disorder in the development of different abilities. The biography research, analyzed four applications available in the technological market highlighting the operation, the objectives, the possibilities and their availability. At the end of the research, a comparative table of the applications was made, evidencing the different characteristics between them. It's possible to conclude that the $A B C$ Autism application has more commercial coverage with more than one hundred thousand downloads and is presented in different versions: English, Portuguese and Spanish. The AUTApp application better addresses the specific needs of children with Autistic Spectrum Disorder such as behavior, emotions, facial expressions and shared attention. The TEO application is distinguished by presenting beyond basic skills, cognitive development activities. Albert's Discoveries App has lower memory capacity and is the only one that can be found on Android and IOS.
\end{abstract}

KEYWORDS: Mobile devices, applications, autismo.

\footnotetext{
* Pedagoga. Psicopedagoga. Especialista em Inclusão Escolar. Mestranda em Educação pelo Programa de Pósgraduação do Ensino da Gestão Básica pela Universidade Federal do Maranhão.

* Doutor em Ciências da Educação com área de especialização em Tecnologia Educativa pela Universidade do Minho (2011), Mestre em Educação Multimídia pela Universidade do Porto (2007), Tecnólogo em Processamento de Dados pelo Centro Universitário UNA (2002) e Licenciado em Pedagogia pela Faculdade do Maranhão (2016). É também Especialista em Docência no Ensino Superior pela PUC-MG (2003), Engenharia de Sistemas pela ESAB (2010) e Educação a Distância pelo UNISEB (2015). Professor Dr $^{\circ}$ vinculado ao Departamento de Educação I do Programa de Pós-graduação do Ensino da Gestão Básica da Universidade Federal do Maranhão

*** Doutorado em Distúrbios do Desenvolvimento pela Universidade Presbiteriana Mackenzie. Mestre em Distúrbios do Desenvolvimento pela Universidade Presbiteriana Mackenzie. Especialista em Psicomotricidade pela Universidade Candido Mendes, Especialista em Intervenção Precoce pela Universidade de Évora, Cursando Especialização em Psicopedagogia Clínica e Institucional, Graduada em Educação Física pela Universidade Federal do Maranhão (UFMA). Graduada em Pedagogia. Membro da Associação Nacional de Pós-graduação e Pesquisa em Educação-ANPED. Professora Adjunta do Departamento de Educação Física da UFMA e do programa de Pós-Graduação Stricto Sensu em Gestão de Ensino da Educação Básica da UFMA. Líder do grupo de pesquisa Educação Especial na Educação Básica. Professora Dr $^{\mathrm{a}}$ vinculada ao Departamento de Educação I do Programa de Pós-graduação do Ensino da Gestão Básica da Universidade Federal do Maranhão.
} 


\section{Introdução}

A inclusão de crianças com Transtorno do Espectro Autista (TEA) tem despertado desafios e dúvidas. A criança com TEA tem o direito de ser educada num ambiente regular, onde a escola proceda às adaptações adequadas no processo de ensino e aprendizagem, a fim de descobrir respostas para o direito de todas terem à educação de qualidade, que considere as suas necessidades. (LOPES, 2011).

Neste aspecto, as tecnologias podem apresentar-se como importantes ferramentas, tanto de uso pessoal quanto educacional para auxiliar no desenvolvimento de crianças com TEA. Dentre as vantagens, encontram-se o acesso à informação de forma rápida, flexível, em tempo real e de qualquer lugar. Isso torna a tecnologia um recurso eficaz no atendimento das necessidades específicas das pessoas com transtornos, ou déficits, como no caso de crianças com Transtorno do Espectro Autista (FERNANDES et al, 2014).

Celulares, smartphones e tablets são considerados responsáveis por romper os limites de tempo e espaço, podendo a aprendizagem acontecer a qualquer hora e em qualquer lugar, dinamizando e otimizando todo o processo educacional (RODRIGUES, 2012).

Tendo em vista a necessidade de encontrar novas metodologias para desenvolver a criança com TEA e utilizando a tecnologia como aliada desse processo, pois entendemos que estas crianças estão intimamente ligadas ao desenvolvimento tecnológico ao qual se encontra exposta essa geração, levantamos o seguinte questionamento: Quais aplicativos podem ser utilizados para atender às necessidades específicas das crianças com Transtorno do Espectro Autista?

Para tanto, justificamos que o interesse em pesquisar sobre tecnologia móvel para auxiliar crianças com TEA, se deve ao fato de uma das investigadoras deste artigo ter atuado como professora de Atendimento Educacional Especializado por 5 (cinco) anos e, recentemente, como tutora de crianças autistas na rede municipal de ensino em São Luís MA, onde utilizávamos constantemente tecnologias como smartphones e tablets por considerá-los recursos necessários para auxiliar no desenvolvimento cognitivo dessas crianças.

Assim, o presente artigo tem como objetivo analisar o uso de quatro aplicativos que podem contribuir para o processo de desenvolvimento de habilidades necessárias às crianças com TEA.Os aplicativos selecionados como base dessa pesquisa são: "ABC Autismo", “AutApp", “TEO” e "As descobertas de Albert". 


\section{Referencial Teórico}

O processo de desenvolvimento de habilidades da criança com TEA precisa ser criteriosamente contemplado com uma relação entre mediação pedagógica, cotidiano e formação de conceitos. As dificuldades encontradas no seu desenvolvimento cotidiano necessitam de estudos e discussões com o intuito de repensar sua educação, dando foco à comunicação e ao comportamento como principais eixos de intervenção pedagógica (ORRÚ, 2012).

É importante aproveitar as habilidades que as crianças desta geração apresentam, como predileção por máquinas fotográficas, celulares, computadores, tablets, jogos, entre outros dispositivos a favor da sua interação, para realizar atividades que promovam o avanço do desenvolvimento infantil. Neste contexto, é possível ver que as tecnologias também proporcionam às crianças com TEA a chance de aprender, criar, pensar e interagir, ajudando a superar suas limitações e valorizar suas potencialidades (ROMERO, 2016).

Quanto mais significativo for o aprendizado para a criança, mais aumentará a oportunidade de promover o seu desenvolvimento e, para tanto, o facilitador deverá ter uma base teórica acerca do TEA de forma que não exiba ideias preconcebidas ou concepções equivocadas (CAMPBELL, 2009, pág. 123).

Para Allan (2015), podem ser utilizados objetos digitais de aprendizagem, vídeos, textos disponíveis na internet, gráficos, mapas virtuais, simulações e animações e aplicativos.

À medida que esses recursos são conhecidos e incorporados como estratégias de ensino, as crianças se envolverão mais. Essas ferramentas contribuem para que conceitos sejam apresentados de uma forma muito mais clara e lúdica, facilitando o desenvolvimento do TEA (ALLAN, 2015, pág. 130).

A autora complementa ainda que os conteúdos interativos podem servir de ponte para a assimilação de informações diversas. Deve-se analisar com cuidado de que forma tais conteúdos realmente auxiliam no desenvolvimento de habilidades para a criança com TEA, sempre levando em conta o contexto e as necessidades do público-alvo. (ALLAN, 2015).

Sobre os processos de aprendizagem dos autistas e as técnicas adequadas para educálos, Rivière (1984) informa:

(...) frequentemente os processos de aprendizagem das crianças autistas são tão lentos e tão alterados que a aplicação rotineira de técnicas educativas termina na frustração se não for acompanhada de uma atitude de indagação ativa e de exploração criativa do que acontece com a pessoa que educamos. Quando acompanhada desta atitude, a relação educativa com crianças autistas (por mais 
exigente que seja) se converte em uma tarefa apaixonante e que pode enriquecer enormemente tanto o professor como o aluno. (RIVIÈRE, 1984, p. 217).

LEBLANC (2015) acredita que é necessário automatizar determinadas intervenções utilizando a tecnologia, o que pode aumentar a precisão e a consistência do ensino das crianças com TEA, podendo também tornar o tratamento mais eficaz, além de reduzir tempo e custos. A psicóloga chama atenção para o fato de que somente quando bem utilizada a tecnologia pode oferecer vantagens no tratamento do comportamento destas crianças. Para ela, é necessário que a tecnologia dos dispositivos eletrônicos permita utilizar também o que há de melhor na tecnologia que é a aplicação prática do conhecimento.

Essa autora aponta, ainda, que é preciso fazer mais duas questões:

Se as necessidades clínicas coincidem com as vantagens que a tecnologia pode oferecer e se temos o conhecimento necessário, tanto da tecnologia em si como da tecnologia do tratamento comportamental. Se a resposta a todas essas perguntas é sim, definitivamente devemos usar a tecnologia (LEBLANC, 2015, online)

O importante é verificar quais características da tecnologia são necessárias para produzir bons efeitos no desenvolvimento destas crianças.

\section{Metodologia}

Este artigo se caracteriza como uma pesquisa bibliográfica, estruturada em três etapas: $1^{\circ}$ ) busca de dados através da loja de aplicativos Google Play, disponível em smartphones com sistema operacional Android. No Google Play, através do descritivo "autismo" foram encontrados 17 aplicativos; destes, foram selecionados quatro para a pesquisa. Como critérios de inclusão foram selecionados apenas os aplicativos que auxiliam na intervenção precoce de crianças com TEA em necessidades básicas como a comunicação, comportamento e atenção compartilhada. Foram excluídos os aplicativos que discutiam sobre processos de alfabetização de crianças com TEA e outros aspectos não relacionados à nossa abordagem. Esta etapa foi realizada no período de março/2018. $2^{\circ}$ ) análise dos aplicativos utilizados na intervenção da criança com TEA. Esta etapa foi realizada no período de março e abril/2018. $\quad 3^{\circ}$ ) análise qualitativa com a realização de um quadro comparativo dos aplicativos pesquisados.

\section{Autismo, tecnologia móvel e aprendizagem}


O Transtorno do Espectro Autista é caracterizado pela presença de um desenvolvimento comprometido ou acentuadamente anormal da interação social e da comunicação e um repertório muito restrito de atividades e interesses, onde podem variar as suas manifestações, dependendo do nível de desenvolvimento e da idade cronológica do indivíduo, com graduação em dois grupos de sintomas: déficit na comunicação e interação social; padrão de comportamentos, interesses e atividades restritos e repetitivos (DSM-5, 2014).

Com a vigência da Lei 12.764/2012, intitulada Lei Berenice Piana, que instituiu a Política Nacional de Proteção dos Direitos da Pessoa com Transtorno do Espectro Autista, fora garantido que "todo autista tem direito à educação e ao ensino profissionalizante", sendo este um passo importante para conhecermos e entendermos como iniciar o processo de ensino e aprendizagem desse aluno, tendo em vista proporcionar-lhes uma educação de qualidade (Política Nacional de Proteção dos Direitos das Pessoas com Transtorno do Espectro Autista, Art. 3º, Parágrafo IV, 2012).

No que se refere à interação social, linguagem e comunicação, compreende-se que sejam feitas intervenções pedagógicas com as crianças com TEA, desenvolvendo e respeitando suas limitações, buscando metodologias inovadoras capazes de auxiliar com motivação a aprendizagem destas crianças.

As propostas pedagógicas de ensino para crianças autistas variam conforme sua necessidade. Entendemos ser necessário desenvolver metodologias apropriadas e variadas dentro dos níveis que apresentam dentro do espectro.

Conforme Silva e Almeida (2012, p. 72), as estratégias pedagógicas direcionadas a alunos autistas devem:

Ampliar a possibilidade de acesso do aluno à linguagem receptiva e expressiva, ampliando assim, o repertório comunicativo do aluno por meio de atividades de vida diária e da comunicação alternativa, visando à autonomia, partindo de seus interesses, respeitando suas possibilidades motoras, cognitivas e afetivas, para promover o avanço conceitual.

Tendo em vista essas possibilidades de práticas educativas e propostas de intervenções no processo de desenvolvimento da criança com TEA, a tecnologia vem se destacando como instrumento de acessibilidade e inclusão, capaz de atender e auxiliar alunos com necessidades educacionais especiais. 
Tem sido no mundo tecnológico que crianças e adolescentes têm descoberto novos caminhos epistemológicos. Neste sentido, observa-se que crianças com TEA aprendem a escrever, a se comunicar e transformam o mundo virtual em um mundo colaborativo, por meio de aplicativos diversos, entre outros recursos (CUNHA, 2012).

Assim, as ferramentas tecnológicas móveis, como o Tablet e o Smartphone, podem ser utilizadas como facilitadoras do processo de ensino e aprendizagem em prol do desenvolvimento destas crianças com TEA.

No universo tecnológico, com a popularização dos smartphones, a explosão mercadológica dos tablets e a emergência dos sistemas automotivos computadorizados, percebe-se uma acessibilidade cada vez maior de um número de usuários. Estes aparelhos possibilitam inúmeras funções, dentre elas o de auxiliar na aprendizagem. Tais dispositivos são cada vez mais inteligentes ( smarts), com interfaces cada vez mais íntimas, imperceptíveis pela familiaridade e naturalidade proporcionada pela prática com que são manuseadas (GOMES, 2016).

Com a disseminação destes dispositivos móveis, observamos a possibilidade de se exercer atividades colaborativas para a aprendizagem, independentemente do lugar e até mesmo em deslocamento (GOMES, 2016).

O tablet é uma das tecnologias mais avançadas atualmente e pode ser extremamente útil no trabalho com autistas. Jogos acessíveis e aplicativos favorecem formas de expressão e comunicação além de se apresentar de modo bem atrativo e de fácil manipulação. A tela sensível ao toque e de fácil uso estimula a concentração e chama atenção com cores e animações (ORRÚ, 2016).

Portanto, a tecnologia móvel, com a utilização de aplicativos disponíveis no mercado tecnológico, é uma oportuna possibilidade para auxiliar as crianças com TEA a aprimorar diferentes habilidades e vivenciar situações desafiadoras de aprendizagem, desenvolvendo o raciocínio lógico, aperfeiçoando a coordenação motora fina e ampla, a percepção visual (tamanho, cor, formas), a percepção auditiva entre outros.

\section{Conhecendo e identificando os aplicativos para crianças com TEA}

A presente pesquisa pôde identificar que já existem no mercado tecnológico diversos aplicativos desenvolvidos para auxiliar crianças com TEA. É vasta a oferta com os mais diversos temas que podem variar de acordo com o interesse e a necessidade da criança. 
Foram selecionados quatro aplicativos. Como critérios de seleção, utilizamos: a gratuidade, a utilização off-line e o auxílio nas necessidades específicas de crianças com TEA tais como: comunicação, comportamento e atenção compartilhada.

Os aplicativos pesquisados têm como objetivos o desenvolvimento de habilidades visuais, transposição de figuras, atividades envolvendo habilidades emocionais (reconhecimento de emoções), reconhecimento das partes do corpo, identificação de números e quantidades, jogo da memória e atividades da vida diária.

Sendo assim, reconhecendo a importância do uso desses aplicativos, compreende-se que poderão auxiliar os profissionais na orientação para estímulos sociais, comunicação e atenção compartilhada. A seguir, apresentamos uma descrição de cada um desses aplicativos:

\section{a) Autismo}

O aplicativo "ABC Autismo" foi desenvolvido pelo Instituto Federal de Alagoas (IFAL), testado pela Associação dos Amigos Autistas (AMA) de Alagoas e adota os princípios do programa Tratamento e Educação para Autistas e Crianças com Déficits relacionados com a Comunicação (TEACCH), criado em 1964 por Eric Schoppler, na Universidade da Carolina do Norte (EUA).

A estrutura do "ABC Autismo" é baseada em quatro níveis de dificuldades, assim como o programa TEACCH. As atividades de habilidades concretas são organizadas e estruturadas visualmente para maior clareza com figuras simples. As crianças fazem desde a transposição de figuras, formas geométricas, letras e sílabas como também formam palavras, identificam vogais e o alfabeto.

O nível 1 são atividades básicas de transposição de figuras, obedecendo a ordem estabelecida no programa TEACCH, da esquerda para a direita. Não há critérios para a imagem, obtendo estas pouco detalhes para evitar a distração do autista. Os elementos usados na área de armazenamento, além de simples, contêm apenas uma única representação para favorecer o transporte de estímulos. $\mathrm{Na}$ área da resposta, há poucos elementos de referência em tamanhos variados de modo a favorecer a resposta correta, inicialmente áreas grandes que diminuem à medida que o autista obtém sucesso na execução da atividade (FARIAS; SILVA; CUNHA, 2014).

As atividades que compõem o Nível 2 visam ao emparelhamento de imagens, das mais diversas formas e cores e exige da criança o discernimento dos elementos para executar as atividades, por meio da diferenciação de cor, tamanho e forma dos elementos da área de 
armazenamento, além de relacioná-los aos existentes na área de resposta. Mais estímulos são dados às crianças, uma vez que terão que se esforçar cognitivamente para definir critérios de discriminação dos elementos entre si, além de selecionar os mesmos corretamente (FARIAS; SILVA; CUNHA, 2014).

O Nível 3 considera que a criança já possui habilidades cognitivas para diferenciação de elementos específicos, exigindo-se que diferencie posturas e ações, bem como realize associações (FARIAS; SILVA; CUNHA, 2014).

No Nível 4 encontramos atividades de composição de palavras, sequenciamento de números e cruzadinhas. É composto por atividades alfabetizadoras, as quais possuem um nível mais elevado de abstração e simbolismo, visando ensinar autistas habilidades básicas de letramento (FARIAS; SILVA; CUNHA, 2014).

O contato com o objeto concreto, realizado de forma convencional no programa TEACCH, é fundamental para a intervenção educacional, não podendo ser deixado de lado, sendo o aplicativo "ABC Autismo" apenas um complemento à dinâmica utilizada no processo de intervenção com a criança. Este aplicativo está disponível gratuitamente para smartphones e tablets com sistema operacional Android na loja Google Play.

Nas figuras dispostas abaixo, mostraremos a tela principal do aplicativo "ABC Autismo" (figura 1) e as entretelas dos quatro níveis do aplicativo (figura 2).

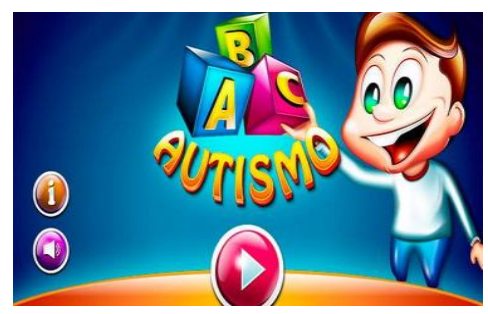

Figura 1 - Tela principal do aplicativo "ABC Autismo"

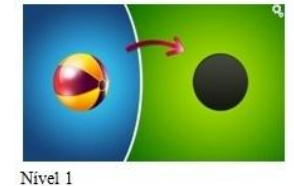

Nivel 1

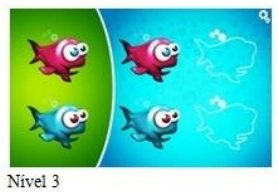

Nivel 3

Figura 2 - Os quatro níveis do aplicativo "ABC Autismo"
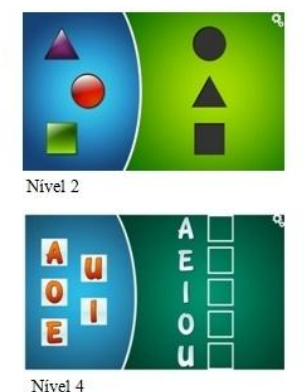


\section{b) AutApp}

É um aplicativo móvel, simples, totalmente gratuito, disponível para smartphones e tablets com sistema operacional Android na loja Google Play. Foi desenvolvido por um projeto de um aluno do Curso de Engenharia da Computação da Faculdade SATC ${ }^{1}$, Gabriel Hahn, que buscou auxiliar de maneira divertida crianças autistas no desenvolvimento da coordenação motora, reconhecimento de cores e formas.

O aplicativo apresenta um personagem chamado Érick, e, a partir dele, trabalha com duas perspectivas: "O que está sentido?" e "Vamos Brincar".

Em “O que está sentindo?”, são realizadas perguntas sobre as emoções que são representadas no rosto do garoto como: triste, feliz, surpreso, raiva.

Em "Vamos Brincar", a aplicativo promove atividades de pareamento/transposição de figuras. A atividade ajuda a estimular o aluno com TEA nos aspectos visual, motor e linguagem.

Abaixo, mostraremos a figura da tela principal do aplicativo AutApp (figura 3) e a tela onde mostra as opções de atividades dispostas no aplicativo (figura 4).

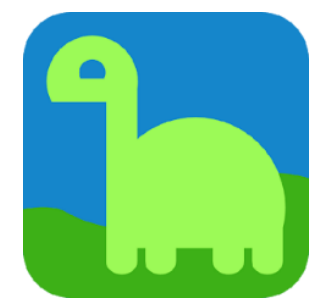

Figura 3 - Tela principal do aplicativo AutApp

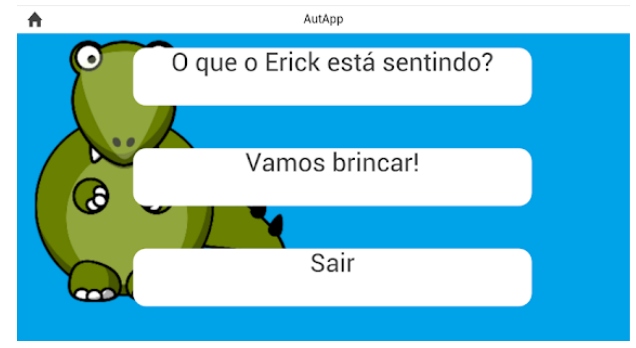

Figura 4 - Atividades propostas no aplicativo AutApp

\footnotetext{
${ }^{1}$ http://www.faculdade.satc.edu.br
} 


\section{c) TEO (Tratar, Estimular e Orientar)}

Desenvolvido pela Universidade Federal de Alagoas (Campos Carapiraca) por um Projeto vinculado ao programa PIBIP-Ação em 2014/2015, o aplicativo TEO traz um conjunto de jogos interativos voltados para crianças com TEA, com objetivo de estimular a comunicação, a socialização e o comportamento das crianças.

A partir de atividades como quebra-cabeça, jogo da memória, jogo de identificação das partes do corpo, transposição de figuras, número/quantidade e atividades da vida diária, o aplicativo auxilia no desenvolvimento de habilidades básicas desde simples tarefas do dia-adia a jogos de raciocínio lógico.

Subdividido em sessões nomeadas: Cores, onde a criança fará a associação das cores através da função touch na figura, fazendo o uso da transposição para unir os pares corretos; Matemática, após fazer a soma das quantidades das figuras, a criança fará a correspondência correta entre número/quantidade; Quebra-cabeça, de forma simples a criança realizará a montagem de figuras como cachorro, menina, peixe e tartaruga; Memória,o jogo medirá a capacidade de memorização e atenção da crianças com TEA; Atividade Diária, nesta modalidade o aplicativo mostra apenas uma atividade, que propõe à criança autista vestir corretamente o menino TEO, colocando nele a roupa adequada a cada parte do corpo; Onde Está?, através de perguntas para identificar a parte do corpo, a criança irá clicar na parte correta.

As atividades propostas no aplicativo têm categorias e deve ser escolhido o nível de dificuldade para a criança brincar.

Aplicativos como este visam capacitar o sujeito autista a interagir socialmente, comunicar-se e desenvolver seu aparato cognitivo.

Totalmente gratuito, pode ser encontrado na Google Play para smartphones e tablets.

A figura abaixo mostra a tela principal do aplicativo TEO (figura 5).

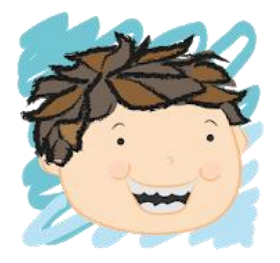

Figura 5 - Tela principal do aplicativo TEO 


\section{d) As descobertas de Albert}

“As descobertas de Albert” é um aplicativo educacional que foi concebido com a missão de ajudar no desenvolvimento de crianças com TEA nos diferentes aspectos em que normalmente elas têm dificuldades, como a capacidade subjetiva e simbólica de utilizar as expressões faciais e verbais para se comunicar.

Tal aplicativo é encontrado gratuitamente para smartphones e tablets com sistema operacional Android na loja Google Play e ainda na configuração do Iphone no App Store.

$\mathrm{Na}$ etapa "olhar", o personagem demonstra através do movimento com os olhos, a direção do objeto ao qual ele quer obter. Na etapa "formas", através da função touch, a criança irá encaixar corretamente as formas geométricas no seu local adequado. Na etapa "facial”, deverá descobrir qual a expressão facial o Albert irá expressar de acordo com o momento vivido por ele. Na etapa "fala", a criança aprenderá qual a forma correta de pedir um objeto a alguém.

A figura abaixo mostra a tela principal do aplicativo "As descobertas de Albert" (figura 6).

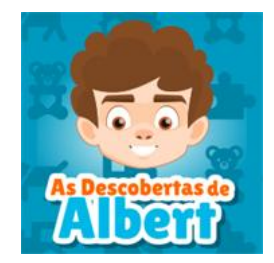

Figura 6 - Tela principal do aplicativo As Descobertas de Albert

\subsection{Sumarizando os aplicativos em análise}

Na Tabela 1 mostramos o quadro descritivo dos aplicativos analisados. Nessa Tabela é demonstrado que os aplicativos pesquisados visam ao desenvolvimento de habilidades necessárias à progressão de alunos com TEA, tais como: comunicação, comportamento e atenção compartilhada. 


\begin{tabular}{|c|c|c|c|c|c|c|c|c|c|c|c|c|}
\hline & $\begin{array}{l}0.0 \\
\text { D. } \\
\text { D. }\end{array}$ & Criação & 莺 & $\begin{array}{l}\frac{n}{\tilde{z}} \\
\frac{0}{1} \\
\vdots \\
0 \\
0\end{array}$ & 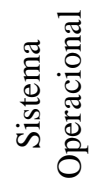 & 节 & 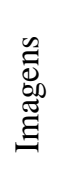 & है & $\stackrel{n}{\Sigma}$ & $\begin{array}{c}\text { Desenvolvimento de } \\
\text { habilidades básicas para } \\
\text { autistas }\end{array}$ & 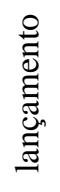 & 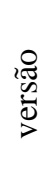 \\
\hline 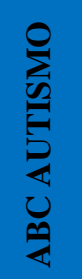 & 总 & AMA & 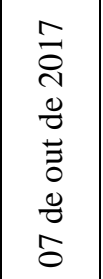 & $\begin{array}{l}8 \\
8 \\
\dot{8} \\
+ \\
+\end{array}$ & $\begin{array}{l}\frac{0}{0} \\
\frac{0}{0} \\
\frac{0}{4}\end{array}$ & 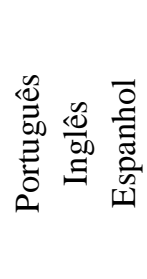 & है & 贯 & $\begin{array}{l}m \\
m \\
\stackrel{g}{g}\end{array}$ & Comportamento & 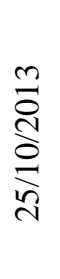 & $\vec{i}$ \\
\hline है & 急 & $\begin{array}{c}\text { Gabriel } \\
\text { HahnSchaeffer, } \\
\text { aluno do curso } \\
\text { de engenharia } \\
\text { da } \\
\text { Universidade } \\
\text { Federal de } \\
\text { Alagoas }\end{array}$ & 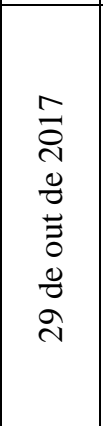 & $\underset{\substack{8 \\
\\
+}}{+}$ & $\begin{array}{l}\frac{0}{0} \\
\frac{0}{0} \\
\frac{0}{4}\end{array}$ & 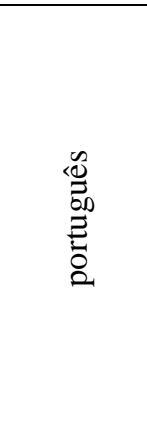 & है & . & ñ. & $\begin{array}{c}\text { Comunicação } \\
\text { Emoções } \\
\text { Visual } \\
\text { Expressões faciais } \\
\text { Atenção } \\
\text { compartilhada }\end{array}$ & 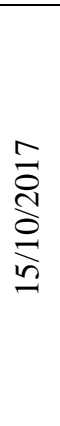 & $\stackrel{n}{0}$ \\
\hline 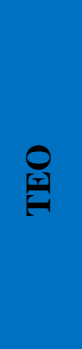 & 总 & $\begin{array}{c}\text { Thiago Bruno } \\
\text { Melo de Sales, } \\
\text { aluno do curso } \\
\text { de engenharia } \\
\text { da } \\
\text { Universidade } \\
\text { Federal de } \\
\text { Alagoas }\end{array}$ & 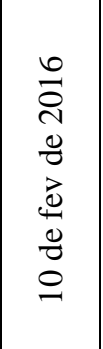 & $\begin{array}{l}8 \\
\vdots \\
0 \\
+ \\
+\end{array}$ & $\begin{array}{l}\frac{0}{0} \\
\frac{0}{0} \\
\frac{0}{0}\end{array}$ & 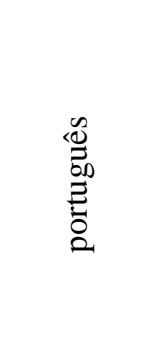 & है & है⿴囗十 & $\begin{array}{l}\infty \\
0 \\
\stackrel{0}{0} \\
\stackrel{0}{0}\end{array}$ & $\begin{array}{c}\text { Comunicação } \\
\text { Interação social } \\
\text { Cognitivo }\end{array}$ & 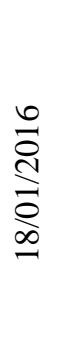 & $\overrightarrow{\text {. }}$ \\
\hline 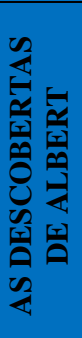 & 莺 & $\begin{array}{l}\text { INOVApps } \\
\text { 2015 - Pablo } \\
\text { Pinheiro }\end{array}$ & 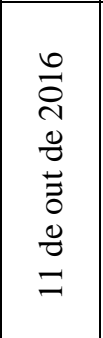 & $\underset{\substack{8 \\
\\
+}}{+}$ & 宽 & 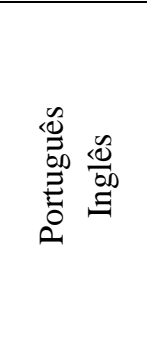 & 贯 & 寻 & 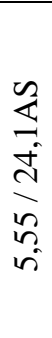 & $\begin{array}{c}\text { Comunicação } \\
\text { Visual } \\
\text { Expressões faciais } \\
\text { Atenção } \\
\text { compartilhada }\end{array}$ & 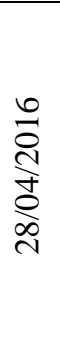 & $\stackrel{\leftrightarrow}{\ddot{0}}$ \\
\hline
\end{tabular}

É possível perceber que o aplicativo “ABC Autismo" tem maior abrangência comercial possuindo mais de 100.000 downloads. Em contrapartida, tem o maior número de $\mathrm{MB}$, ocupando maior espaço na memória do celular. O número muito grande de downloads se deve ao fato de ser encontrado em versões no Português, Inglês e Espanhol, o que o difere dos demais aplicativos. 
O aplicativo "AutApp" destaca-se entre os demais por desenvolver uma habilidade específica relacionada às emoções. As crianças com TEA não apresentam sinais de sentir e perceber afetivamente o outro e, por isso, atividades como estas podem auxiliar no desenvolvimento dessa particularidade. Apresenta uma configuração pequena de apenas 9,37 MB, ocupando pouca memória do celular.

O aplicativo "TEO”, além de desenvolver habilidades básicas da criança com TEA, diferencia-se por apresentar atividades relacionadas à aprendizagem escolar desenvolvendo o cognitivo através de jogos de raciocínio lógico, matemática, jogo da memória e quebracabeça.

O aplicativo "As Descobertas de Albert” diferencia-se dos demais por ser o único apresentado nas versões Android e IOS, podendo assim ser utilizado em smartphones e Iphone, Tablets e Ipad.

\section{Considerações finais}

O uso da tecnologia no processo de aprendizagem é permeado pela ideia de que é preciso estender as possibilidades de aplicações dos recursos tecnológicos ao ensino de pessoas com autismo. A dinâmica dos jogos atrelados à tecnologia móvel chama a atenção de forma diferenciada, propiciando um elo entre as habilidades necessárias para o desenvolvimento destas crianças e o uso da tecnologia.

Os aplicativos descritos nesta pesquisa podem ser utilizados para atender crianças com TEA no desenvolvimento de habilidades específicas que estas crianças não possuem, como, por exemplo, o comportamento, a comunicação, as emoções, as expressões e a atenção compartilhada. Uma vez aliados a um mesmo dispositivo o som, a imagem, o design e a interatividade, o aplicativo possibilita à criança novas maneiras de estimular a aprendizagem.

É necessário que se priorizem investimentos na área da Educação Especial, capacitando os profissionais para o uso da tecnologia como recurso para o desenvolvimento de crianças com TEA e que estes estejam abertos a buscar as funcionalidades que as novas tecnologias podem proporcionar. 
Como trabalhos futuros, espera-se aplicar estes aplicativos com diversas crianças que apresentam Transtorno do Espectro Autista, a fim de se perceber de fato as mudanças sociais que elas poderão promover. Segundo Mello e Sganzerla (2013) e Farias e Cunha (2015) o uso destes aplicativos em crianças autistas pode ajudar no desenvolvimento das habilidades motoras, na autonomia, na diminuição das barreiras de contato com outros indivíduos. Os autores afirmam que tais aplicativos ainda podem ajudar a criança com esse tipo de transtorno a se expressar melhor, além de possibilitar ganhos em aspectos cognitivos e no raciocínio lógico.

Findamos a nossa pesquisa com o intuito de ter auxiliado profissionais que lidam com esse público na seleção dos aplicativos, frisando que a escolha destes está atrelada às necessidades específicas de cada criança com TEA.

\section{Referências bibliográficas}

BRASIL, Lei $\mathrm{n}^{\mathrm{o}}$ 12.764, de 27 de dezembro de 2012. Disponível em: < http://www.planalto.gov.br/ccivil_03/_ato2011-2014/2012/lei/112764.htm> Acesso em: 23/04/2018.

CUNHA, E. Autismo e Inclusão: psicopedagogia e práticas educativas na escola e na família. Rio de Janeiro: Wak Editora, 2012.

FARIAS, E. B. ; SILVA, L. W. C. ; CUNHA, M. X. C. . ABC AUTISMO: Um aplicativo móvel para auxiliar na alfabetização de crianças com autismo baseado no Programa TEACCH. In: X Simpósio Brasileiro de Sistemas de Informação, 2014, Londrina - PR. Anais do 10o Simpósio Brasileiro de Sistemas de Informação, 2014. Disponível em <http://goo.gl/5swXXf $>$, acesso em 20 Mar 2018.

FARIAS, E. B.; CUNHA, M. X. C. ; SOUZA, J. W. S. ABC Autismo? Um a Aplicação Mobile para Auxiliar no Processo Alfabetizador de Crianças com Autismo. In: IV Congresso Brasileiro de Informática na Educação, 2015, Maceió. Anais dos Workshops do IV Congresso Brasileiro de Informática na Educação (CBIE 2015), 2015.

FERNANDES, F. G.; OLIVEIRA, L. C. de; RODRIGUES, Mylene Lemos; VITA, Stéfano Schwenck Borges Vale. (2014).

GOMES, C. Smartphones e Tablets: ferramentas para expandir a sala de aula. Curitiba: Appris, 2016.

LOPES, M. T. V. Inclusão das crianças autistas. Dissertação (Mestrado em Ciências da Educação) - Universidade de Lisboa. Orientador: Nuno Mateus. Lisboa, 2011. 193 f. 
Manual diagnóstico e estatístico de transtorno: DSM-5 / [American Psychiatnc Association, traduç . Maria Inês Corrêa Nascimento ... et al.]; revisão técnica: Aristides Volpato Cordioli... [et al.]. - . e . Porto Alegre: Artmed, 2014. xliv, 948 p.; 25 cm.

MELlO, C. M. C.; SGANZERLA, Maria Adelina Raupp. Proposta de aplicativo Android para auxiliar no desenvolvimento matemático de pessoas com autismo. In: VI Congresso Internacional de Ensino da Matemática, 2013, Canoas. VI Congresso Internacional de Ensino da Matemática, 2013.

ORRÙ, S. E. Aprendizes com autismo: aprendizagem por eixo de interesses em espaços não excludentes. Petrópoles, RJ: Vozes, 2016.

ORRÚ, S. E. Austimo, linguagem e educação: interação social no cotidiano escolar. Rio de Janeiro: Wak Editora, 2012.

RIVIÈRE, A. Modificacion de Conducta em el Autismo Infantil. Revista Espanõla de Pedagogia, v. XLII, p. 164-5, 1984.

RODRIGUES, C. Universidade Trocam Livros por Tablets em Cursos a Distancia. Disponível em: <https://portal.aprendiz.uol.com.br/arquivo/2012/05/02/universidades-trocamlivros-por-tablets-em-cursos-a-distancia/> Acesso em 21 março 2018.

ROMERO, P. O aluno autista: avaliação, inclusão e mediação. Prefácio de Eugênio Cunha. Rio de Janeiro: Wak Editora, 2016.

SILVA, F. da S. ALMEIDA, A. L. de. Atendimento educacional especializado para alunos com autismo: desafios e possibilidades. Intl. J. ofKnowl. Eng., Florianópolis, v. 1, n. 1, p. 62 $-88,2012$. 